\title{
Integrated workforce capacity and inventory management under labour supply uncertainty
}

\section{Fazil Pac , Osman Alp \& Tarkan Tan}

To cite this article: M. Fazil Pac , Osman Alp \& Tarkan Tan (2009) Integrated workforce capacity and inventory management under labour supply uncertainty, International Journal of Production Research, 47:15, 4281-4304, DOI: 10.1080/00207540801930237

To link to this article: http://dx.doi.org/10.1080/00207540801930237

$$
\text { 曲 Published online: } 15 \text { May } 2009 .
$$

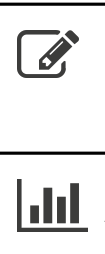

Submit your article to this journal $\pi$

川 Article views: 200

Q View related articles $\asymp$

7 Citing articles: 10 View citing articles 


\title{
Integrated workforce capacity and inventory management under labour supply uncertainty
}

\author{
M. Fazil Pac ${ }^{\mathrm{a}}$, Osman Alp ${ }^{\mathrm{b} *}$ and Tarkan $\operatorname{Tan}^{\mathrm{c}}$ \\ ${ }^{a}$ Operations and Information Management Department, The Wharton School, University of \\ Pennsylvania, Philadelphia, PA, USA; ${ }^{b}$ Industrial Engineering Department, Bilkent University, \\ Bilkent, Ankara, Turkey; ' Department of Technology Management, Eindhoven University of \\ Technology, Eindhoven, The Netherlands
}

(Received 18 January 2007; final version received 26 October 2007)

\begin{abstract}
In a manufacturing environment with volatile demand, inventory management can be coupled with dynamic capacity adjustments for handling the fluctuations more effectively. In this study, we consider the problem of integrated capacity and inventory management under non-stationary stochastic demand and capacity uncertainty. The capacity planning problem is investigated from the workforce planning perspective where the capacity can be temporarily increased by utilising contingent workers from an external labour supply agency. The contingent capacity received from the agency is subject to an uncertainty, but the supply of a certain number of workers can be guaranteed through contracts. There may also be uncertainty in the availability of the permanent and contracted workers due to factors such as absenteeism and fatigue. We formulate a dynamic programming model to make the optimal capacity decisions at a tactical level (permanent workforce size and contracted number of workers) as well as the operational level (number of workers to be requested from the external labour supply agency in each period), integrated with the optimal operational decision of how much to produce in each period. We analyse the characteristics of the optimal policies and we conduct an extensive numerical analysis that helps us provide several managerial insights.
\end{abstract}

Keywords: inventory; capacity management; flexible capacity; workforce availability; supply uncertainty

\section{Introduction}

Production and capacity decisions of manufacturing firms are significantly affected by demand volatility. In some industries, dynamic capacity adjustments arise as an effective tool for handling this volatility. The production capacity can be temporarily increased by acquiring external capacity resources such as outsourcing, renting machinery, hiring contingent workers, etc. Effective utilisation of such resources results in increased demand responsiveness and reduced operational costs. However, external capacity may not always be available at the desired quantity and/or quality in the environment. Therefore, the uncertainty of external capacity supply should be considered in production planning.

*Corresponding author. Email: osmanalp@bilkent.edu.tr 
In this study, we consider the integrated planning of production/inventory and capacity under demand and external capacity supply uncertainties.

Capacity can be defined as the maximum amount of production that can be achieved by utilising internal and external resources, whereas capacity flexibility stands for the ability to change the capacity temporarily. Especially when the inventory holding and/or backordering costs are high, capacity flexibility may prove to be an efficient tool for meeting the volatile demand. We consider labour-intensive manufacturing environments and hence we consider capacity in terms of the workforce. Throughout the text, we use the terms 'workers' and 'capacity' interchangeably. We classify the production capacity under two main categories: permanent capacity and contingent capacity. Permanent capacity is formed by the company's own workforce under a steady payroll, whereas the contingent capacity is formed by the workers that can be acquired temporarily from an external labour supply agency (ELSA). In practice, availability of both capacity types may be subject to uncertainty. Permanent capacity may fail to be exercised in full terms due to absenteeism, sickness, fatigue, etc. Similarly, a manufacturer's request for contingent workers may be totally or partially unmet by the ELSA due to factors such as size of available temporary worker pool, capability of finding skilled workers, competition in the environment, demand structure of different customers, and opportunities in alternative options. In the case where there is a high demand for contingent workers in the market at the time of the request, or if the manufacturer requires the workers at short notice, the risk of the request not being met in full terms is higher. Moreover, ELSAs may not be willing to fulfill a specific request at a specific time, considering potentially better options. Therefore, the availability of contingent workers may be a major concern when the manufacturer relies on external capacity for production.

The uncertainty of capacity availability can be expressed as either the 'allor-nothing type' or 'partial availability type'. Under the former type, either all or none of the requested capacity is acquired with corresponding probabilities, whereas the requested amount may be acquired partially under the latter type. In practice, the probability of acquiring any given amount of capacity may depend on the amount requested. For example, a possible partial availability type could be a case where ELSA is more likely to fulfill the manufacturer's request when relatively lower capacity levels are requested.

A labour supply contract between the manufacturer and the ELSA is a possible way of alleviating the impacts of labour supply uncertainty on the manufacturer where the manufacturer pays a certain fee per contracted worker per period (reservation cost), and the ELSA is committed to provide the required number of workers up to the contracted quantity to the manufacturer with certainty with an additional fee per worker requested (utilisation cost). Note that this type of contracting is known as 'option contracting'. The manufacturer may still request temporary workers in addition to the contracted workers, but the supply of those workers is subject to uncertainty. Under this setting, we classify contingent workers under two categories: contracted workers and temporary workers.

Dynamic adjustments of the permanent capacity, such as hiring or firing, are generally too costly. For example, firing workers brings in direct compensation costs, which are substantial in many countries, especially in Europe. Similarly, hiring permanent workers generally requires a costly thorough search, which also entails mismatch risks. Moreover, such adjustments tend to have negative effects on the efficiency of workers due to the social and motivational effects. Therefore, we consider the determination of the permanent 
capacity level as a tactical decision that is made at the beginning of a planning horizon and not changed until the end of it. Utilising flexible capacity is a means of overcoming these issues, and we consider this as one of the two main operational tools of coping with fluctuating demand, along with holding inventory. Consequently, the decisions that we consider are the determination of the permanent workforce size and the number of contracted workers from the ELSA at the beginning of the planning horizon, as well as the number of temporary workers to request from the ELSA and the production quantity in each period.

Although our motivation for this problem originated from a labour-intensive manufacturing environment, a similar framework could also fit environments where capacity is in other terms. For example, additional production capacity could be acquired from a subcontractor whose production capacity is also demanded by other manufacturers, creating an uncertainty in the availability of the subcontractor's capacity, which can be decreased by reserving some of this capacity.

There exists a significant usage of a flexible workforce in many countries. For example, $6.6 \%$ of the active labour force of the Netherlands was composed of flexible workers (temporary, standby, replacement, and other such workers) in 2003 (Beckers 2005). The US Bureau of Labor Statistics (2006) reports that, in February 2005, there were 14.8 million flexible workers (independent contractors, on-call workers, temporary help agency workers, and workers provided by contract firms) constituting $10.7 \%$ of total employment in the USA. $11.9 \%$ of these workers are related to manufacturing. Aside from the workers with alternative work arrangements as indicated above, contingent workers accounted for $4.1 \%$ of total US employment. In March 2006, 7.9\% of the active labour force in Turkey was composed of contingent workers (Turkish Statistical Institute 2006). Contingent workers can be hired anytime and are generally paid for labour hours. The productivity of contingent workers may vary for industries requiring different skill levels, with the productivity loss increasing in skill requirements.

\section{Literature review}

Capacity planning has been analysed extensively at all levels of decision making. An in-depth review, presenting the formulation and solution of strategic capacity problems, is provided by Van Mieghem (2003). Holt et al. (1960) pioneered the research in the field of workforce planning and flexibility, with their seminal work analysing the trade-off between keeping large permanent workforce levels and frequent capacity adjustments. Our model considers the same problem in essence, extending it to the case of demand and capacity supply uncertainty. Wild and Schneeweiss (1993) analyse manpower capacity planning with a hierarchical approach using stochastic dynamic programming.

In a particularly relevant work, Milner and Pinker (2001) consider the problem of designing labour supply contracts between firms and ELSAs under demand and temporary labour supply uncertainty. The authors consider a single-period setting where the supply uncertainty is either in terms of productivity loss or unavailability. In the former case, if the labour request that is placed after demand materialisation exceeds the contracted quantity, it is met with certainty by the ELSA at a higher cost. In the latter case, the unavailability is a function of the number of temporary workers available in the 
market and the fee the firm pays to the ELSA per temporary worker. In our work, we focus on the firm's problem under a particular contract type. We consider a multiperiod setting and focus on several unavailability structures of labour supply. Moreover, in our model, capacity decisions are made before the demand materialises, which implicitly takes the supply lead time into account since it can be considered that contingent workers are requested at the end of the previous period. Another disparity is that we consider a production environment including the option of inventory carrying, whereas they consider a service environment.

The particular contract type that we consider is known as 'option contracting' in the literature. There are two decision variables for the buyer in an option contracting setting: the number of options to be bought and the number of options to be exercised depending on the state of the system. By buying options in advance, the buyer provides incentives for the supplier to build capacity. This setting is partially similar to our problem, as the manufacturer signs a contract with ELSA to guarantee the supply of capacity under a certain cost to be paid even when contracted workers are not utilised. However, in our problem setting we also consider a further layer of flexibility by requesting additional capacity, the supply of which is subject to uncertainty and its utilisation is to be decided according to materialisation. Barns-Schuster et al. (2002) analyse option contracts in a two-period environment under correlated demand and show that option contracting mitigates the operational costs. Cachon (2003) presents an extensive survey of the literature on option contracts, as well as other type of contracts, used in supply chain environments. Main stream research on option contracting deals with determining the contract parameters in a game theoretic environment where both parties have conflicting objectives. Together with the differences in the particular problem environment that we consider, the major difference between our work and this stream of research is that we focus on the effect of a given option contract on the operating decisions in a multiple-period setting. Tan (2004) analyses an environment with a capacitated producer and a subcontractor. The availability of the subcontractor is subject to uncertainty; however, a level of availability is guaranteed by a contract. The author shows that a threshold policy with two bands is optimal. This policy is similar to the optimal production policy of our environment under no supply uncertainty and under a particular uncertainty structure.

Of the papers that consider integrated production and capacity planning, the following are relevant to our work. Pinker and Larson (2003) consider the problem of managing permanent and contingent workforce levels under uncertain demand where inventory holding is not allowed. The sizes of regular and temporary labour are decision variables that are fixed throughout the planning horizon, but the capacity level may be adjusted by setting the number of shifts for each class of workers. Dellaert and de Kok (2004) investigate the integrated flexible capacity and production planning problem considering a production capacity composed of long-term contract workers and temporary workers. The approach of planning capacity and production in an integrated manner outperforms the decoupled approach. Hu et al. (2004) also investigate an integrated flexible capacity and production planning problem on a continuous-time framework under Markovmodulated demand. In a similar problem, Tan and Gershwin (2004) study production and subcontracting strategies with limited production capacity and fluctuating demand, considering lead time sensitive customers. Atamturk and Hochbaum (2001) focus on the integrated capacity and production planning problem under a non-stationary deterministic demand setting exploiting the trade-offs between capacity expansions, subcontracting and 
carrying inventory. Angelus and Porteus (2002) present a simultaneous capacity and production planning problem for short life-cycle products, considering capacity expansions as well as contractions. Yang et al. (2005) consider a production/inventory system under Markovian internal capacity levels and an outsourcing option, where the outsourcing decision is made for the next period after observing the realised capacity and the demand. The authors characterise the optimal production and outsourcing decisions under the assumption that the outsourcing option is fully reliable, whereas we assume uncertainty in contingent capacity in our model.

Our work is closely related to the problems considered by Tan and Alp (2005), Mincsovics et al. (2006), and Alp and Tan (2007). These three papers consider settings similar to ours, ignoring labour supply uncertainty. Tan and Alp (2005) focus on the operational decisions under the existence of fixed costs for initiating production and for using contingent capacity. Alp and Tan (2007) extend this analysis by including the tactical level decision of determining the permanent capacity levels. Finally, Mincsovics et al. (2006) model and analyse the problem under a lead time associated with the acquisition of contingent capacity.

Considering the field of production/inventory planning under random capacity/yield, Yano and Lee (1995) provide an extensive review of the literature on lot sizing under random production or procurement yields. Ciarallo et al. (1994) analyse the optimality of extended myopic policies under uncertain capacity and uncertain demand in a periodic review setting. In their setting, the production decision is made prior to the realisation of the random capacity. In a later work, Iida (2002) shows that the problem introduced by Ciarallo et al. (1994) is equivalent to determining the production quantities after the realisation of the random capacity. The major difference in our model is that we consider a more general case where the capacity acquisition probabilities may depend on requested capacity. Hence, we deal with an integrated capacity and production planning problem, whereas they consider a production planning problem under capacity uncertainty. This leads to the necessity of defining two separate decision variables in our model, one for the requested capacity and the other for the production decision after the capacity is realised, which should be optimised simultaneously, and hence their optimality results cannot be generalised to our case.

Kouvelis and Milner (2002) analyse the joint effects of demand and supply uncertainty on capacity and outsourcing decisions in multi-stage supply chains. The authors indicate that, as the supply uncertainty increases, capacity investments increase. Unlike our model, they do not consider operational production/inventory decisions. In another related work, Schmitt and Snyder (2006) consider a system with supply disruptions under all-or-nothing availability. The environment that we model is different to a 'material supply uncertainty' environment such as that of Schmitt and Snyder (2006), because we have the option of not utilising some of the capacity that is acquired, which is not the case when material supply is considered. Moreover, we also consider several partial availability structures.

\section{The model}

We first present our basic definitions, assumptions and settings in Section 3.1. Then we provide the dynamic programming formulation in Section 3.2, which can be used to solve the integrated capacity and inventory management problem under consideration. Finally, we consider the single-period problem in Section 3.3. 


\subsection{Problem settings}

\subsubsection{Capacity availability and uncertainty}

We consider a labour-intensive manufacturing environment where the manufacturer employs a fixed number of permanent workers, $U$, throughout a given planning horizon of length $T$. The production capacity can be increased temporarily by hiring temporary workers from ELSAs. The availability of temporary workers is subject to uncertainty. As a measure to guard against this uncertainty, the manufacturer has the option to make a contractual agreement with ELSA so that ELSA guarantees to supply a contracted number of temporary workers - denoted by $V$ - whenever requested. There may also be uncertainty in the availability of the permanent and contracted workers due to absenteeism, fatigue, etc. While our model can accommodate all kinds of uncertainty structures, ELSA is contractually required to provide the contracted workers whenever requested, therefore we do not consider any uncertainty in the availability of the contracted workers. We treat $U$ and $V$ as decision variables to be determined at the beginning of the planning horizon by the manufacturer. At the beginning of each period $t=1, \ldots, T$, the inventory level $x_{t}$ is observed, and a capacity target, $w_{t}$, is determined. The capacity target corresponds to the total number of workers requested. If $w_{t} \leq U$, no contingent workers are requested, if $U \leq w_{t} \leq U+V$, then contracted workers are requested in addition to the permanent workers, and, finally, if $U+V \leq w_{t}$, then the manufacturer requests all contracted workers and some extra temporary workers from ELSAs. However, in any of these cases, the requested capacity target $w_{t}$ may not be acquired in full due to the uncertainty in capacity availability. The amount of capacity acquired in period $t, m_{t}$, depends on the requested quantity, $w_{t}$, with a probability function of $P_{t}\left(m_{t}, w_{t}\right)$, which we refer to as the availability function. This function is the convolution of the availability function of permanent capacity, $P_{t}^{\mathrm{p}}\left(m_{t}^{\mathrm{p}}, w_{t}^{\mathrm{p}}\right)$, and that of temporary capacity, $P_{t}^{\mathrm{tw}}\left(m_{t}^{\mathrm{tw}}, w_{t}^{\mathrm{tw}}\right)$. We assume that this uncertainty structure is known by the manufacturer exactly.

The availability function $P_{t}\left(m_{t}, w_{t}\right)$, which is a joint probability function, can be used to express any form of uncertainty in capacity availability. For example, if we assume that the permanent and contracted capacity are fully available with certainty and, in the case where temporary capacity is requested, acquiring any amount up to the requested capacity is equally likely, then we have

$$
P_{t}\left(m_{t}, w_{t}\right)= \begin{cases}0, & \text { if } m_{t}<w_{t} \leq U \text { or } m_{t}<U+V \leq w_{t}, \\ \frac{1,}{\left(w_{t}-U-V\right)}, & \text { if } m_{t}=w_{t} \leq U+V, \\ \text { if } U+V<m_{t} \leq w_{t} \text { or } U+V=m_{t}<w_{t} .\end{cases}
$$

Note that, in this specific example,

$$
P_{t}^{\mathrm{p}}\left(m_{t}^{\mathrm{p}}, w_{t}^{\mathrm{p}}\right)= \begin{cases}1, & \text { if } m_{t}^{\mathrm{p}}=w_{t}^{\mathrm{p}} \leq U \\ 0, & \text { otherwise }\end{cases}
$$

and

$$
P_{t}^{\mathrm{tw}}\left(m_{t}^{\mathrm{tw}}, w_{t}^{\mathrm{tw}}\right)= \begin{cases}\frac{1}{w_{t}^{\mathrm{tw}}}, & \text { if } m_{t}^{\mathrm{tw}} \leq w_{t}^{\mathrm{tw}} \\ 0, & \text { otherwise. }\end{cases}
$$


Moreover, there could be an upper limit on the number of temporary workers requested, where any number above this limit cannot be acquired. Such situations can also be handled in our model by considering appropriate availability functions.

\subsubsection{Cost structure}

Once $m_{t}$ is observed, a production decision $Q_{t} \leq m_{t}$ is made to raise the inventory level to $y_{t}=x_{t}+Q_{t}$. At the end of the period, the demand $z_{t}$ is realised and met. Demand in period $t, Z_{t}$, has distribution function $G_{t}(z)$. The remaining inventory is carried to the next period at a cost of $h$ per unit and any unmet demand is fully backordered at a unit cost of $b$ per period. We assume that there are no shortages of raw material and the lead time of production and acquiring external capacity can be neglected. There are no fixed costs for initiating production and no material-related costs are considered in the model. The notation is summarised in Table 1. Further explanation of notation will be provided as need arises.

The cost of permanent capacity is independent of the production quantity and paid each period even if there is no production. The unit cost of permanent capacity is $c_{\mathrm{p}}$ per period. Therefore, the total permanent capacity cost for a workforce of size $U$ is $c_{\mathrm{p}} U$ per period. In the particular contract type that we consider, each contracted worker costs $\hat{c}_{\mathrm{r}}$ per period, independent of the utilisation. There is also an additional cost component $\hat{c}_{\mathrm{u}}$ for each contracted worker utilised in production per period. Consequently, the cost of a utilised contracted worker per period, $\hat{c}_{\mathrm{cw}}$, is $\hat{c}_{\mathrm{cw}}=\hat{c}_{\mathrm{r}}+\hat{c}_{\mathrm{u}}$. Also, let $\hat{c}_{\mathrm{tw}}$ be the cost of a hired temporary worker per period. In order to synchronise the production quantity with the number of workers, we redefine the 'unit production' as the number of actual units that an average permanent worker can produce per period. We also define the cost of

Table 1. Summary of notation.

\begin{tabular}{ll}
\hline$T$ & Number of periods in the planning horizon \\
$U$ & Size of available permanent capacity \\
$V$ & Size of available contracted capacity \\
$c_{\mathrm{p}}$ & Unit cost of permanent capacity per period \\
$c_{\mathrm{r}}$ & Unit reservation cost of contracted capacity per period \\
$c_{\mathrm{u}}$ & Unit utilisation cost of contracted capacity per period \\
$c_{\mathrm{cw}}$ & Total unit cost of contracted capacity $\left(c_{\mathrm{cw}}=c_{\mathrm{r}}+c_{\mathrm{u}}\right)$ \\
$c_{\mathrm{tw}}$ & Unit cost of temporary capacity per period \\
$h$ & Inventory holding cost per unit per period \\
$b$ & Penalty cost per unit of backorder per period \\
$\alpha$ & Discounting factor $(0<\alpha \leq 1)$ \\
$w_{t}$ & Capacity requested in period $t$ \\
$m_{t}$ & Capacity acquired in period $t$ \\
$Q_{t}$ & Number of items produced in period $t$ \\
$Z_{t}$ & Random variable denoting the demand in period $t$ \\
$G_{t}(z)$ & Distribution function of $Z_{t}$ \\
$P_{t}\left(m_{t}, w_{t}\right)$ & Probability function of receiving $m_{t}$ workers when $w_{t}$ workers are requested \\
$x_{t}$ & Inventory position at the beginning of period $t$ before production \\
$y_{t}$ & Inventory position in period $t$ after production \\
$f_{t}\left(x_{t}, U, V\right)$ & Minimum total expected cost of operating the system in periods $t, t+1, \ldots, T$, \\
& given the system state $x_{t}, U$, and $V$ \\
\hline
\end{tabular}


production by contingent workers on the same unit basis, where the cost for contingent workers is related to their productivity. Consequently, the term ' $w$ workers are requested' stands for requesting workers that are sufficient to produce $w$ units. Considering that productivity rates of permanent, contracted and temporary workers may differ, let $\lambda_{\mathrm{cw}}$ and $\lambda_{\text {tw }}$ be the average productivity rates of contracted workers and temporary workers, respectively, relative to the productivity of permanent workers. The model is valid for all values of $\lambda_{\mathrm{cw}}>0$ and $\lambda_{\mathrm{tw}}>0$, however it is likely that $0<\lambda_{\mathrm{tw}} \leq \lambda_{\mathrm{cw}} \leq 1$. Assuming that the productivity rates remain approximately unchanged in time, the unit production cost by contracted workers, $c_{\mathrm{cw}}$, can be written as $c_{\mathrm{cw}}=\hat{c}_{\mathrm{cw}} / \lambda_{\mathrm{cw}}$, where the production-equivalent unit reservation and utilisation costs by contracted workers, $c_{\mathrm{r}}$ and $c_{\mathrm{u}}$, are $c_{\mathrm{r}}=\hat{c}_{\mathrm{r}} / \lambda_{\mathrm{cw}}$ and $c_{\mathrm{u}}=\hat{c}_{\mathrm{u}} / \lambda_{\mathrm{cw}}$, respectively. Hence, the total reservation cost of contracted workers is $c_{\mathrm{r}} V$ for a total contracted capacity of $V$ production units. Similarly, the unit production cost by temporary workers, $c_{\mathrm{tw}}$, can be written as $c_{\mathrm{tw}}=\hat{c}_{\mathrm{tw}} / \lambda_{\mathrm{tw}}$. The total cost of temporary workers is $c_{\mathrm{tw}} m_{t}$ if the firm observes $m_{t}$ temporary workers, regardless of whether they are utilised or not.

\subsection{Dynamic programming formulation}

The minimum cost of operating the system from period $t$ until the end of the planning horizon is denoted by $f_{t}\left(x_{t}, U, V\right)$, where we drop $U$ and $V$ from the notation for brevity whenever appropriate. While the availability functions can be probability mass functions (i.e. defined on a discrete domain), we consider them to be continuous for generalisation purposes to cover cases such as the workers leaving production at any time due to sickness, etc. or some capacity environments other than the labour force. We assume an ending condition of $f_{T+1}=0$. We model our integrated capacity and inventory management problem as follows:

$$
\begin{aligned}
f_{t}\left(x_{t}, U, V\right) & =\min _{w_{t} \geq 0}\left\{H_{t}\left(w_{t} \mid x_{t}, U, V\right)\right\}, \quad \text { for } t=1,2, \ldots, T, \\
f_{0}\left(x_{1}\right) & =\min _{U \geq 0, V \geq 0}\left\{f_{1}\left(x_{1}, U, V\right)\right\},
\end{aligned}
$$

where

$$
H_{t}\left(w_{t} \mid x_{t}, U, V\right)=g_{t}\left(w_{t} \mid U, V\right)+\int_{0}^{w_{t}}\left[\varphi_{t}\left(m_{t} \mid x_{t}\right)\right] P_{t}\left(m_{t}, w_{t}\right) \mathrm{d} m_{t} .
$$

Here, $g_{t}\left(w_{t} \mid U, V\right)$ denotes the expected total cost of capacity in period $t$, for given $U$ and $V$ values. In our problem environment this function can be stated as

$$
\begin{aligned}
g_{t}\left(w_{t} \mid U, V\right)= & U c_{\mathrm{p}}+V c_{\mathrm{r}} \\
& + \begin{cases}0, & \text { if } 0 \leq w_{t} \leq U, \\
\left(w_{t}-U\right) c_{\mathrm{u}}, & \text { if } U \leq w_{t} \leq U+V, \\
V c_{\mathrm{u}}+\int_{0}^{w_{t}-U-V} m_{t} c_{\mathrm{tw}} P_{t}^{\mathrm{tw}}\left(m_{t}^{\mathrm{tw}}, w_{t}-U-V\right) \mathrm{d} m_{t}^{\mathrm{tw}}, & \text { if } U+V \leq w_{t} .\end{cases}
\end{aligned}
$$

The function $\varphi_{t}\left(m_{t} \mid x_{t}\right)=\min _{y_{t}: x_{t} \leq y_{t} \leq x_{t}+m_{t}}\left\{L_{t}\left(y_{t}\right)+\alpha E\left[f_{t+1}\left(y_{t}-z_{t}\right)\right]\right\}$ denotes the production decision function that attains the minimum total expected cost of operations in periods $t, t+1, \ldots, T$, excluding the immediate labour costs, where $L_{t}\left(y_{t}\right)=$ $h \int_{0}^{y_{t}}\left(y_{t}-z_{t}\right) \mathrm{d} G_{t}(z)+b \int_{y_{t}}^{\infty}\left(z_{t}-y_{t}\right) \mathrm{d} G_{t}(z)$ is the regular convex loss function. 


\subsection{Single-period problem}

Now we consider the last period problem. Let $\hat{y}_{T}$ be the minimiser of $L_{T}\left(y_{T}\right)$ and $y_{T}^{*}$ be the optimal inventory level after production under an acquired capacity of $m_{T}$ in the last period. Then we have

$$
y_{T}^{*}= \begin{cases}x_{T}+m_{T}, & \text { if } x_{T}+m_{T} \leq \hat{y}_{T} \\ \hat{y}_{T}, & \text { if } x_{T} \leq \hat{y}_{T} \leq x_{T}+m_{T}, \\ x_{T}, & \text { if } \hat{y}_{T}<x_{T},\end{cases}
$$

and

$$
\varphi_{T}\left(m_{T} \mid x_{T}\right)= \begin{cases}L_{T}\left(x_{T}+m_{T}\right), & \text { if } x_{T}+m_{T} \leq \hat{y}_{T}, \\ L_{T}\left(\hat{y}_{T}\right), & \text { if } x_{T} \leq \hat{y}_{T} \leq x_{T}+m_{T}, \\ L_{T}\left(x_{T}\right), & \text { if } \hat{y}_{T}<x_{T} .\end{cases}
$$

Then substituting (2) into (1), we obtain the following cost function for requesting $w_{T}$ workers in total, for $x_{T} \leq \hat{y}_{T}$ :

$$
\begin{aligned}
H_{T}\left(w_{T} \mid x_{T}\right)= & g_{T}\left(w_{T} \mid U, V\right)+\int_{0}^{\hat{y}_{T}-x_{T}} L\left(x_{T}+m_{T}\right) P_{T}\left(m_{T}, w_{T}\right) \mathrm{d} m_{T} \\
& +\int_{\hat{y}-x_{T}}^{w_{T}} L\left(\hat{y}_{T}\right) P_{T}\left(m_{T}, w_{T}\right) \mathrm{d} m_{T} .
\end{aligned}
$$

This implies that if the optimal quantity to produce, $\hat{y}_{T}-x_{T}$ is less than or equal to the acquired capacity, $m_{T}$, then it is optimal to produce up to $\hat{y}_{T}$, leaving a portion of the available capacity unutilised. We note that this property would hold for any period $t$, if $L_{t}\left(y_{t}\right)+\alpha E\left[f_{t+1}\left(y_{t}-z_{t}\right)\right]$ was convex, which we observe not to hold in general in our numerical experiments that were conducted on a discrete space.

While the uncertainty structures of different capacity sources can be analysed using the model presented, our focus in the rest of this paper is on the uncertainty of the temporary capacity.

\section{All-or-nothing-type temporary capacity availability}

In all-or-nothing-type uncertainty, the firm receives $w_{t}$ temporary workers with probability $p$ and does not receive any worker with probability $(1-p)$. In particular, we have

$$
P_{t}\left(m_{t}, w_{t}\right)= \begin{cases}p, & \text { if } U+V<w_{t}=m_{t}, \\ 1-p, & \text { if } U+V=m_{t}<w_{t}, \\ 1, & \text { if } m_{t}=w_{t} \leq U+V, \\ 0, & \text { otherwise. }\end{cases}
$$

This may happen, for example, when the ELSA has better offers from other firms and therefore rejects the offer of the firm. Here $1-p$ can be considered as the probability of ELSA having better alternatives. It may also be the case, for example in assembly lines, that while the ELSA is able to supply the firm's request partially, such a partial supply is not acceptable to the firm. 
In this section we characterise the structure of the optimal policy for the all-or-nothing case for given $U$ and $V$. The following theorem characterises the optimal inventory and capacity management policy when $p$ is reasonably large $\left(p \geq c_{\mathrm{u}} / c_{\mathrm{tw}}\right)$. Relatively low values of $p$ would not be sustainable for the operations anyway, since a certain reliability of ELSA is necessary.

Theorem 4.1: If $p \geq c_{u} / c_{t w}$, then (i) the multi-period decision function $H_{t}\left(w_{t} \mid x_{t}\right)$ is convex in $w_{t}$, (ii) the optimal production policy is of state-dependent order-up-to type and the optimal order-up-to level can be stated as

$$
y_{t}^{*}\left(x_{t}\right)= \begin{cases}y_{t c}^{*}, & \text { if } x_{t}<y_{t c}^{*}-U-V, \\ x_{t}+U+V, & \text { if } y_{t c}^{*}-U-V<x_{t} \leq y_{t v}^{*}-U-V, \\ y_{t v}^{*}, & \text { if } y_{t v}^{*}-U-V<x_{t} \leq y_{t v}^{*}-U, \\ x_{t}+U, & \text { if } y_{t v}^{*}-U<x_{t} \leq y_{t p}^{*}-U, \\ y_{t p}^{*}, & \text { if } y_{t p}^{*}-U<x_{t} \leq y_{t p}^{*}, \\ x_{t}, & \text { if } y_{t p}^{*}<x_{t},\end{cases}
$$

where $y_{t p}^{*}, y_{t v}^{*}$ and $y_{t c}^{*}$ are three critical numbers that are independent of the starting inventory levels for each period $t$, and they refer to production with permanent capacity only, production with permanent and contracted capacity only, and production with permanent, contracted and temporary capacity, respectively, and (iii) the optimal capacity ordering decision is given by $w_{t}^{*}\left(x_{t}\right)=y_{t}^{*}\left(x_{t}\right)-x_{t}$.

Proof: See Appendix A.

Corollary 4.2: In the special case of $V=0, H_{t}\left(w_{t} \mid x_{t}\right)$ is convex in $w_{t}$ for all $x_{t}$ and $t$.

Theorem 4.1 states that the optimal production decision determines the capacity ordering decision, meaning that the problem reduces to one with a single decision variable. Note that this result simplifies the problem significantly. When the starting inventory level is low and use of temporary workers is required for production, the optimal number of temporary workers to be requested is as many as necessary for materialising the optimal production quantity. The acquired capacity level is fully used for production irrespective of whether or not all of the temporary workers ordered are received. Finally, we present the following theorem which proves that, as the probability of success increases, the optimal order-up-to level, and hence the optimal capacity requested, have a non-increasing structure.

Theorem 4.3: The optimal order-up-to level $y_{t}^{*}\left(x_{t}\right)$ is non-increasing in the probability of success $p$.

Proof: See Appendix A.

This theorem indicates that, as the ELSA becomes more reliable, the temporary workers requested from the ELSA decrease.

\section{Partial temporary capacity availability}

Partial availability can be modelled in various forms depending on the operating characteristics of the ELSAs. We model and analyse the following forms of partial 
availability: (i) uniform availability where the firm has an equally likely chance of acquiring 0 to $w$ workers; (ii) normal availability where the number of workers to be received is distributed approximately with a (discrete) Normal distribution; (iii) decreasing availability where the ELSA has a limited temporary worker pool size, $K$, and a relatively stable market so that as $w$ increases, the probability of acquiring each worker decreases; (iv) moderate availability where lower demands are not preferred by the ELSA in order to prevent the temporary worker pool size from shrinking, while higher demands have a lower chance of being met due to the scarcity of supply; (v) increasing availability where the ELSA attempts to avoid the division of its workforce for this purpose and tries to satisfy larger-sized requests to a great extent, (vi) high-low availability where the ELSA wants to meet larger and smaller-sized requests and deter from committing a moderate size of its workers to a firm, considering the chance of larger-sized demands from other customers. These structures are summarised in Table 2 .

We analyse these partial availability cases mainly based on numerical analysis, as they are analytically intractable. In the case of uniform supply uncertainty, we show that the last period's cost function is convex in the requested capacity target $w$ for a certain condition on cost coefficients, while the multi-period cost function is observed to be non-convex. Under other uncertainty types, we observe that the problem is non-convex both in single- and multi-period cases. Therefore, the search for the optimal solution is conducted in an exhaustive manner. While we presented our model as a finite horizon model, our numerical results are conducted for the case of $T \rightarrow \infty$ to facilitate computations. In the problem settings we consider, we observe that the solution of the finite horizon problem converges to that of the infinite horizon problem rapidly. The algorithm is coded in $\mathrm{C}$ programming language. We denote the (discrete) normal availability case with a coefficient of variation of $\mathrm{CoV}$ as Normal[CoV]. We truncated the supply probabilities exceeding the range $[0, w]$ by adding the summation of the out-ofrange probabilities to the corresponding bound, in constructing the normal availability. We take $K=20$ in the availability structures with a binomial distribution. We consider Poisson demand where the mean demand is taken as 10 in Section 5.1 and the demand

Table 2. Summary of capacity availability structures considered.

\begin{tabular}{llr}
\hline Availability structure & \multicolumn{1}{c}{ Distribution } & Pool size \\
\hline $\begin{array}{l}\text { Uniform } \\
\text { Normal } \\
\text { Decreasing }\end{array}$ & $\begin{array}{l}\text { Discrete uniform }(0, w) \\
\text { Discrete normal }\left(w / 2, \sigma^{2}\right) \\
\text { Binomial }(w, p=\max \{K-w, 0\} / K)\end{array}$ & $\begin{array}{c}\text { Unlimited } \\
\text { Unlimited } \\
K\end{array}$ \\
Moderate & Binomial $\left(w, p=\left\{\begin{array}{ll}\frac{\cos (2 \pi w / K-\pi)+1}{2}, & \text { if } w \leq K, \\
0, & \text { otherwise }\end{array}\right)\right.$ & $K$ \\
Increasing & Binomial $(w, p=\min \{w, K\} / K)$ & $K$ \\
High-Low & Binomial $\left(w, p=\left\{\begin{array}{ll}\frac{\sin (2 \pi w / K+\pi / 2)+1}{2}, & \text { if } w \leq K, \\
0, & \text { otherwise }\end{array}\right)\right.$ & $K$ \\
\hline
\end{tabular}


following a seasonal pattern with a cycle of four periods, the expected demand being 10 , 15, 10, and 5, respectively, in Section 5.2, unless otherwise noted. We drop subscript $t$ when we refer to an infinite horizon solution. Similarly, we consider a stationary labour supply uncertainty distribution function, $P(m, w)$. In the results that we present, we use the term 'increasing' ('decreasing') in the weak sense to mean 'non-decreasing' ('non-increasing'). We provide intuitive explanations for all our results below and our findings are verified through several numerical studies. However, like any experimental result, one should be careful about generalising them, especially for extreme values of problem parameters.

\subsection{Optimal production and capacity ordering policies}

In this section we provide an analysis of the cost functions, $f_{t}\left(x_{t}\right)$ and $H_{t}\left(w_{t} \mid x_{t}\right)$, and the characteristics of the optimal production and capacity ordering policies for different forms of supply availabilities. Our numerical analysis on the discrete space shows that $f_{t}\left(x_{t}\right)$ is not necessarily convex. However, in all problem instances that we solved, this function is quasiconvex. On the other hand, the decision function, $H_{t}\left(w_{t}\right)$, is not necessarily (quasi-)convex, as can be observed from Figure 1 for an infinite horizon problem instance. The violating behaviour is observed around $w=20$ where the probability of success is low under the particular high-low availability structure considered. Nevertheless, we show that the last period's decision function is convex under uniform availability when $c_{\mathrm{tw}}$ is at least $2 c_{\mathrm{u}}$.

Theorem 5.1: Under Uniform availability, the last period's decision function $H_{T}\left(w_{T} \mid x_{T}\right)$ is convex for all $x_{T}$ when $c_{u} \leq c_{t w} / 2$.

Proof: See Appendix A.

Corollary 5.2: In the special case of $V=0, H(w \mid x)$ is convex for all $x$ under uniform availability.

In all availability structures considered, there exists a threshold starting inventory level value, below which temporary workers are utilised in addition to the 'ensured' (permanent

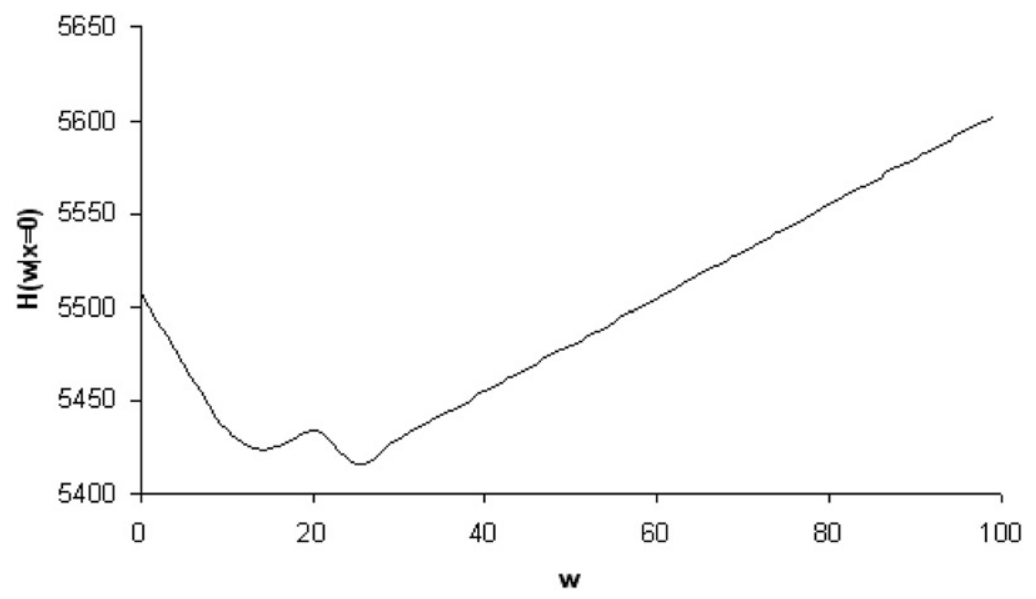

Figure 1. $H(w \mid x=0)$ vs. requested capacity - high-low availability, $c_{\mathrm{p}}=2.5, c_{\mathrm{tw}}=3.5, h=1$, $b=5, U=10$. 
plus contracted) capacity, and above which only ensured capacity is utilised. As the inventory level, $x$, increases above this threshold, use of contracted and subsequently permanent capacity is curtailed. In this region, the following capacity usage for production is optimal: (i) all ensured capacity; (ii) all permanent workers and a portion of the contracted workers; (iii) all of the permanent workers; (iv) a portion of the permanent workers, and (v) none. Cases (ii) and (iv) correspond to two distinct order-up-to levels. For the special case of no temporary workers, Tan and Alp (2005) prove that this policy is indeed optimal. However, when $x$ is below this threshold the optimal production decision depends on a particular realisation of the capacity acquired, which is a random variable. Therefore, under supply uncertainty the expectation of $y^{*}(x), E\left[y^{*}(x)\right]$, does not necessarily follow an order-up-to-type behaviour, which is shown to be optimal by Tan and Alp (2005) for deterministic supply.

As pointed out in Section 3, not every worker that is paid for, even a temporary one, is utilised in the optimal solution. The decision maker sets the requested capacity target considering the expected outcome, consequently the production decision is made according to the acquired capacity. We call the difference between the acquired capacity level and the optimal production level the surplus of temporary capacity (STC). We examine the effect of availability structure on the STC. Uniform availability yields the highest STC among all availability structures. This is because the Uniform distribution has no peak around any point, i.e. it is platykurtic (has negative kurtosis). Therefore, no particular region of the availability function has more likelihood than the rest, and hence the system cannot request capacity in a way that matches the required production quantity with precision. In a particular problem instance, the total expected STC under uniform availability is nine times more than that under normal availability. The STC values in increasing, decreasing, high-low, and moderate availability structures are very close to zero since the requested capacity target is set to a value which produces a high success probability of acquiring the desired capacity level value.

\subsection{Sensitivity analysis}

In this section we investigate the impact of labour supply uncertainty, demand variability, and cost parameters on operational and tactical decisions. Unless otherwise noted we let $h=1, c_{\mathrm{p}}=2.5, c_{\mathrm{r}}+c_{\mathrm{u}}=3, c_{\mathrm{tw}}=3.5$, and $\alpha=0.99$. In some of our experiments, we assume normal demand with $\mathrm{CoV}$ values of $0.1,0.2$, and 0.3 and Gamma demand with $\mathrm{CoV}$ values of $0.5,1.0$, and 1.5 , to investigate the effect of demand variability on flexible capacity management.

\subsubsection{Effect of labour supply uncertainty}

In this section we investigate the effects of labour supply uncertainty on flexible capacity and production management. Table 3 illustrates the change in average inventory level and the contribution of temporary workers in production under deterministic labour supply, and normal and uniform availability structures. The average inventory level carried increases as we switch from deterministic labour supply to uncertain supply. Under uniform availability, the average inventory levels carried increase drastically when $U=6$. This is because the probability of acquiring low capacity levels is much higher when compared with other availability structures and the system tries to avoid backorders originating from this by holding higher inventory levels. Nevertheless, this is not the case 
Table 3. Comparison of supply structures. $U=6, V=0$.

\begin{tabular}{|c|c|c|c|c|c|c|c|}
\hline \multirow[b]{2}{*}{ Parameters } & \multirow[b]{2}{*}{ Criteria } & \multicolumn{3}{|c|}{$U=6, V=0$} & \multicolumn{3}{|c|}{$U=10, V=2$} \\
\hline & & Deterministic & $\begin{array}{c}\text { Normal } \\
{[0.15]}\end{array}$ & Uniform & Deterministic & $\begin{array}{c}\text { Normal } \\
{[0.15]}\end{array}$ & Uniform \\
\hline $\begin{array}{l}c_{\mathrm{tw}}=1.5 \\
b=50\end{array}$ & $\begin{array}{l}\text { Ave. Inv. Lev. } \\
\text { \% Temporary }\end{array}$ & $\begin{array}{r}7.38 \\
40.88\end{array}$ & $\begin{array}{r}7.99 \\
41.22\end{array}$ & $\begin{array}{l}11.34 \\
40.3\end{array}$ & $\begin{array}{l}7.44 \\
7.52\end{array}$ & $\begin{array}{l}7.59 \\
8.49\end{array}$ & $\begin{array}{l}8.35 \\
5.07\end{array}$ \\
\hline $\begin{array}{l}c_{\mathrm{tw}}=4.5 \\
b=50\end{array}$ & $\begin{array}{l}\text { Ave. Inv. Lev. } \\
\% \text { Temporary }\end{array}$ & $\begin{array}{r}7.58 \\
40.04\end{array}$ & $\begin{array}{r}8.22 \\
40.05\end{array}$ & $\begin{array}{l}12.01 \\
40.05\end{array}$ & $\begin{array}{l}8.73 \\
2.59\end{array}$ & $\begin{array}{l}8.80 \\
2.9\end{array}$ & $\begin{array}{l}9.20 \\
1.88\end{array}$ \\
\hline
\end{tabular}

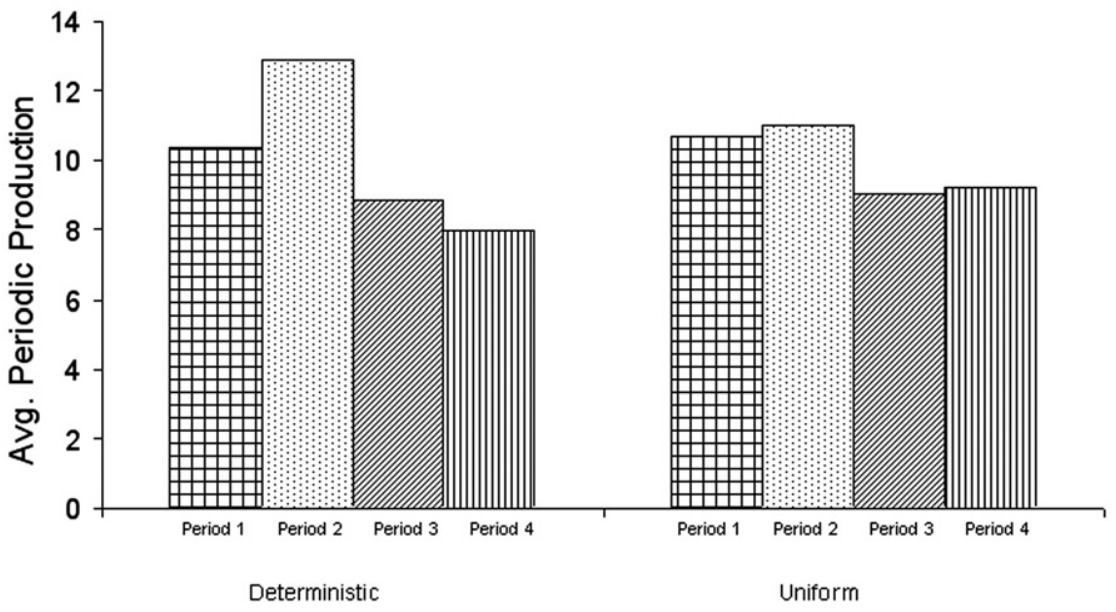

Figure 2. Periodic production-deterministic supply vs. uniform availability. $U=10, V=0$, $c_{\mathrm{tw}}=3.5, b=50$.

when $U=10$ and $V=2$, since the system depends less on the temporary workers in this case. Finally, the average production made with temporary workers is not affected much by the different problem parameters considered when $U=6$, since the low permanent capacity level is almost always fully utilised anyway.

When the level of ensured capacity is sufficient to produce the average demand and the temporary labour supply has high variability, we observe that the manufacturer spreads the total production among periods, rather than utilising flexible capacity against the demand seasonality (see Figure 2).

\subsubsection{Optimal contracted capacity level}

In this section we analyse the effects of the problem parameters $c_{\mathrm{r}}, c_{\mathrm{u}}, c_{\mathrm{tw}}, b$, labour supply uncertainty, and demand uncertainty on the optimal size of the contracted capacity for a given permanent capacity. This analysis provides insights into the number of contingent workers to contract when the manufacturer operates with a suboptimal permanent capacity level. Table 4 depicts the optimal contracted capacity size under different labour 
Table 4. Optimal contracted capacity level $\left(V^{*}\right) . U=6, c_{\mathrm{tw}}=3.5$.

\begin{tabular}{llccccc}
\hline$b$ & $c_{\mathrm{r}}$ & Normal $[0.1]$ & Normal $[0.15]$ & Normal $[0.2]$ & Normal $[0.25]$ & Uniform \\
\hline 2.5 & 0.6 & 3 & 4 & 4 & 4 & 5 \\
2.5 & 1.2 & 2 & 2 & 2 & 3 & 4 \\
2.5 & 1.8 & 2 & 2 & 2 & 2 & 3 \\
2.5 & 2.4 & 2 & 2 & 2 & 2 & 3 \\
2.5 & 3 & 2 & 2 & 2 & 2 & 3 \\
5.5 & 0.6 & 3 & 4 & 4 & 4 & 5 \\
5.5 & 1.2 & 2 & 2 & 2 & 2 & 4 \\
5.5 & 1.8 & 2 & 2 & 2 & 2 & 3 \\
5.5 & 2.4 & 1 & 2 & 2 & 2 & 3 \\
5.5 & 3 & 1 & 3 & 4 & 4 & 7 \\
50 & 0.6 & 3 & 2 & 2 & 2 & 5 \\
50 & 1.2 & 1 & 1 & 2 & 2 & 3 \\
50 & 1.8 & 1 & 1 & 1 & 2 & 3 \\
50 & 2.4 & 1 & 1 & 1 & 2 & 2 \\
50 & 3 & 1 & & 2 & & 3 \\
\hline
\end{tabular}

supply uncertainty structures. First of all, as the reservation cost, $c_{\mathrm{r}}$ becomes larger, naturally $V^{*}$ decreases. Nevertheless, even when $c_{\mathrm{r}}=c_{\mathrm{cw}}$ (which makes the contracted capacity practically equivalent to the permanent capacity), we observe that keeping contracted workers may still be beneficial depending on other cost parameters. As the labour supply uncertainty increases, the system prefers contracting higher capacities, as expected. In the normal availability cases, the system carries higher safety stock to avoid backorders as the backordering cost increases. This leads to system's preference in higher capacity flexibility in order to avoid idle capacity costs. However, since all system parameters interact in the optimal decisions, this result cannot be generalised. For example, the uncertainty of the labour supply in the uniform availability dominates this affect and the system prefers higher contracted capacity levels in order to decrease temporary workers usage, as discussed before.

The effect of demand variability on the optimal contracted capacity heavily interacts with cost parameters. In the Normal availability structure, $V^{*}$ decreases as the demand variability increases when $c_{\mathrm{tw}}$ is not much larger than $c_{\mathrm{cw}}$, as illustrated in Table 5 for $c_{\mathrm{tw}}=3.5$, in order to avoid unutilised contracted capacity. On the other hand, when $c_{\mathrm{tw}}$ is significantly larger than $c_{\mathrm{cw}}$, the opposite behaviour is observed since the system tries to avoid using expensive temporary labour, as illustrated in Table 5 for $c_{\mathrm{tw}}=7.5$. However, the labour supply uncertainty structure also plays an important role in this interaction. For example, in the increasing availability structure, the system reserves higher contracted capacity as the demand variability increases since acquiring a small number of workers from the ELSA is not probable.

\subsubsection{Optimal permanent and contracted capacity decisions}

In this section we investigate the optimal levels of permanent and contracted capacity under various settings. Table 6 illustrates the effect of temporary labour cost and labour supply uncertainty on the optimal capacity levels (permanent and contracted). We observe that, as the labour supply uncertainty increases, the level of ensured capacity also increases in line with our observation in Section 5.2.2. When there is no labour supply uncertainty, 
Table 5. Effect of demand uncertainty on optimal contracted capacity level. $U=4, b=50$.

\begin{tabular}{|c|c|c|c|c|c|c|c|c|}
\hline \multirow[b]{2}{*}{ Labour supply } & \multirow[b]{2}{*}{$c_{\mathrm{tw}}$} & \multirow[b]{2}{*}{$c_{\mathrm{r}}$} & \multicolumn{6}{|c|}{ Demand distribution } \\
\hline & & & $\begin{array}{c}\text { Normal } \\
{[0.1]}\end{array}$ & $\begin{array}{c}\text { Normal } \\
{[0.2]}\end{array}$ & $\begin{array}{c}\text { Normal } \\
{[0.3]}\end{array}$ & $\begin{array}{c}\text { Gamma } \\
{[0.5]}\end{array}$ & $\begin{array}{c}\text { Gamma } \\
{[1.0]}\end{array}$ & $\begin{array}{c}\text { Gamma } \\
{[1.5]}\end{array}$ \\
\hline Normal [0.25] & 3.5 & $\begin{array}{l}0.6 \\
1.2 \\
1.8\end{array}$ & $\begin{array}{l}8 \\
6 \\
6\end{array}$ & $\begin{array}{l}8 \\
5 \\
5\end{array}$ & $\begin{array}{l}7 \\
5 \\
4\end{array}$ & $\begin{array}{c}6 \\
4 \\
3\end{array}$ & $\begin{array}{l}4 \\
2 \\
2\end{array}$ & $\begin{array}{l}2 \\
0 \\
0\end{array}$ \\
\hline Normal [0.25] & 7.5 & $\begin{array}{l}0.6 \\
1.2 \\
1.8\end{array}$ & $\begin{array}{r}10 \\
9 \\
7\end{array}$ & $\begin{array}{r}10 \\
10 \\
8\end{array}$ & $\begin{array}{r}11 \\
10 \\
8\end{array}$ & $\begin{array}{r}11 \\
10 \\
9\end{array}$ & $\begin{array}{r}12 \\
11 \\
9\end{array}$ & $\begin{array}{l}13 \\
12 \\
10\end{array}$ \\
\hline Increasing & 3.5 & $\begin{array}{l}0.6 \\
1.2 \\
1.8\end{array}$ & $\begin{array}{l}8 \\
6 \\
6\end{array}$ & $\begin{array}{l}9 \\
7 \\
6\end{array}$ & $\begin{array}{l}9 \\
8 \\
7\end{array}$ & $\begin{array}{l}8 \\
7 \\
6\end{array}$ & $\begin{array}{r}11 \\
10 \\
8\end{array}$ & $\begin{array}{r}11 \\
10 \\
8\end{array}$ \\
\hline
\end{tabular}

Table 6. Effect of temporary labour cost and uncertainty on ensured capacity $\left(U^{*}, V^{*}\right) . c_{\mathrm{p}}=2.5$, $c_{\mathrm{r}}=0.6, b=50$.

\begin{tabular}{lccccc}
\hline$c_{\mathrm{tw}}$ & Norm [0.1] & Norm [0.15] & Norm [0.2] & Norm [0.25] & Uniform \\
\hline 2.5 & $(2,0)$ & $(3,0)$ & $(4,0)$ & $(5,0)$ & $(8,3)$ \\
3.5 & $(8,0)$ & $(8,1)$ & $(8,1)$ & $(8,1)$ & $(8,4)$ \\
\hline
\end{tabular}

Alp and Tan (2007) show that, for $c_{\mathrm{p}}=c_{\mathrm{tw}}$, the optimal permanent capacity level is zero. Nevertheless, this does not turn out to be the case under labour supply uncertainty in order to hedge against this uncertainty. Moreover, it may be optimal to reserve contracted capacity even when the cost of a contracted worker is higher than that of a temporary worker, when the supply uncertainty is high, as is the case under uniform availability with $V^{*}=3$. Finally, we note that the ensured capacity level increases as $c_{\mathrm{tw}}$ increases, as expected.

\section{Conclusions}

In this study, we consider the problem of integrated capacity and inventory management under non-stationary stochastic demand and capacity uncertainty. We investigate the problem under the workforce planning framework. The focus of the paper is modelling and analysing the effects of temporary labour uncertainty. We model a number of possible availability structures for this purpose: all-or-nothing, uniform, normal, decreasing, increasing, moderate, and high-low. Our model and analysis provide insights into the optimal usage of all capacity means coupled with inventory management in this environment. At the tactical level, these means are contracting a number of contingent workers whose availability is ensured by a reservation cost and determining the optimal level of permanent capacity. At the operational level, the decisions to make are determining the number of workers to be requested from the external labour supply agency and the quantity of production in each period. 
We show for the all-or-nothing-type availability that the resulting cost function is convex under a reasonable condition and the optimal production policy is of statedependent order-up-to type, which dictates the capacity ordering decision. In the case of uniform supply uncertainty, we show that the last period's cost function is convex in the requested capacity target for a certain condition on the cost coefficients, while the multiperiod cost function is observed to be non-convex. Under other uncertainty types, we observe that the cost function is non-convex both in single- and multi-period cases. Furthermore, requesting capacity in such a way that the expected acquired capacity mimics the optimal requested capacity of the deterministic capacity availability case is suboptimal.

We also show that not every temporary worker that is paid for is utilised in the optimal solution. Such a surplus of temporary capacity is highest for the uniform availability structure, followed by the normal availability structure. The surpluses in increasing, decreasing, high-low, and moderate availability structures are very close to zero. We observe that uniform availability performs worst among all availability structures that we considered in all of our experiments. This is because the uniform distribution is platykurtic (has negative kurtosis). The absence of any 'peak' in the uniform distribution makes it difficult to manage this availability structure, especially when a larger number of workers are required. Increasing, decreasing, high-low and moderate availability structures are easier to manage, since the requested capacity target can be set to a value that produces a high success probability of acquiring the desired capacity level in those cases. In the normal availability case, the performance deteriorates as the variability increases.

Our analysis provides insights into the number of contingent workers to contract for any given permanent capacity level. In particular, even when the reservation cost constitutes $100 \%$ of the contracted worker cost, we observe that keeping contracted workers may still be beneficial. Moreover, we also provide managerial insights when the permanent capacity level can be optimised as well as the contracted capacity. Even when the cost of temporary labour is higher than that of permanent or contracted labour, it may still be the case that the optimal or contracted capacity level is positive in order to hedge against supply uncertainty.

This research can be extended by considering the perspective of the external labour supply agency. In such a context, the optimal capacity planning of the ELSA and the contract design problem between the ELSA and the manufacturer might be of interest. Investigating the implications of the manufacturer's contract design preferences on the ELSA and resolving issues such as how the risks would be shared by both parties are among further research questions.

\section{References}

Alp, O. and Tan, T., 2007. Tactical capacity management under capacity flexibility in make-to-stock systems. IIE Transactions, 40 (3), 221-237.

Angelus, A. and Porteus, E.L., 2002. Simultaneous capacity and production management of shortlife-cycle, produce-to-stock goods under stochastic demand. Management Science, 48 (3), 399-413.

Atamtürk, A. and Hochbaum, D.S., 2001. Capacity acquisition, subcontracting, and lot sizing. Management Science, 47 (8), 1081-1100. 
Barnes-Schuster, D., Bassok, Y., and Anupindi, R., 2002. Coordination and flexibility in supply contracts with options. Manufacturing \& Service Operations Management, 4 (3), 171-207.

Beckers, I., 2005. Fewer flexible workers. Web Magazine. Available from http://www.cbs.nl/en-gb/ menu/themas/arbeid-inkomen-sociale-zekerheid/ arbeidsmarkt/publicaties/artikelen/2005-1616wm.htm [Accessed 9 March 2006].

Cachon, G.P., 2003. In: A.G. de Kok and S.C. Graves, eds. Supply chain coordination with contracts. Vol. 11, Amsterdam: Elsevier: Handbooks in OR \& MS, 229-339.

Ciarallo, F.W., Akella, R., and Morton, T.E., 1994. A periodic review, production planning model with uncertain capacity and uncertain demand-optimality of extended myopic policies. Management Science, 40 (3), 320-332.

Dallaert, N. and de Kok, T., 2004. Integrating resource and production decisions in a simple multistage assembly system. International Journal of Production Economics, 90, 281-294.

Holt, C.C., et al., 1960. Planning production, inventories and workforce. Englewood Cliffs, NJ: Prentice-Hall.

Hu, J.Q., Vakili, P., and Huang, L., 2004. Capacity and production management in a single product manufacturing system. Annals of Operations Research, 125, 191-204.

Iida, T., 2002. A non-stationary periodic review production-inventory model with uncertain production capacity and uncertain demand. European Journal of Operational Research, 140, 670-683.

Kouvelis, P. and Milner, J.M., 2002. Supply chain capacity and outsourcing decisions: the dynamic interplay of demand and supply uncertainty. IIE Transactions, 34, 717-728.

Milner, J.M. and Pinker, E.J., 2001. Contingent labor contracting under demand and supply uncertainty. Management Science, 47 (8), 1046-1062.

Mincsovics, G., Tan, T., and Alp, O., 2006. Integrated capacity and inventory management with capacity acquisition lead times. Beta Technical Report WP 191. Technische Universiteit Eindhoven.

Pac, M.F., 2006. Integrated flexible capacity and inventory management under flexible capacity uncertainty. Thesis (MSc). Bilkent University.

Pinker, E.J. and Larson, R.C., 2003. Optimizing the use of contingent labor when demand is uncertain. European Journal of Operational Research, 144, 39-55.

Schmitt, A. and Snyder, V.L., 2006. Infinite-horizon models for inventory control under yield uncertainty and disruptions. Department of Industrial and Systems Engineering. Lehigh University.

Tan, B., 2004. Subcontracting with availability guarantees: production control and capacity decisions. IIE Transactions, 36 (8), 711-724.

Tan, B. and Gershwin, S.B., 2004. Production and subcontracting strategies for manufacturers with limited capacity and volatile demand. Annals of Operations Research, 125, 205-232.

Tan, T. and Alp, O., 2005. An integrated approach to inventory and flexible capacity management subject to fixed costs and non-stationary stochastic demand. OR Spectrum, to appear, DOI: $10.1007 / \mathrm{s} 00291-008-0122-y$.

Turkish Statistical Institute, 2006. Labour force survey, Available from http://www.turkstat.gov.tr [Accessed 20 June 2006].

US Bureau of Labor Statistics, 2006. Contingent and alternative employment arrangements. Available from http://www.bls.gov/cps [Accessed 10 June 2006].

Van Mieghem, J.A., 2003. Capacity management, investment, and hedging: review and recent developments. Manufacturing \& Service Operations Management, 5 (4), 269-302.

Wild, B. and Shneeweiss, C., 1993. Manpower capacity planning - a hierarchical approach. International Journal of Production Economics, 30/31, 95-106.

Yang, J., Qi, X., and Xia, Y., 2005. A production-inventory system with Markovian capacity and outsourcing option. Operations Research, 53 (2), 328-349.

Yano, C.A. and Lee, H.L., 1995. Lot sizing with random yields: a review. Operations Research, 42 (2), 311-334. 


\section{Appendix A: Proofs of theorems}

Proof (Proof of Theorem 4.1): We begin by proving the convexity of the last (single) period cost function, $H_{T}(w \mid x)$. In all-or-nothing availability, $H_{T}(w \mid x)$ can be rewritten by suppressing the subscript $T$ as follows:

$$
H(w \mid x)= \begin{cases}\min _{y: x \leq y \leq x+w}\{L(y)\}, & \text { if } 0 \leq w \leq U, \\ (w-U) c_{\mathrm{u}}+\min _{y: x \leq y \leq x+w}\{L(y)\}, & \text { if } U<w \leq U+V, \\ V c_{\mathrm{u}}+p\left(c_{\mathrm{tw}}(w-U-V)+\min _{y: x \leq y \leq x+w}\{L(y)\}\right) & \\ \quad+(1-p) \min _{y: x \leq y \leq x+U+V}\{L(y)\}, & \text { if } U+V<w .\end{cases}
$$

Let $\hat{y}$ be the minimiser of the convex function $L(y)$, which is known to be $G^{-1}(b /(h+b))$ from the classical newsvendor solution. Note that when $\hat{y} \leq x$ we have $\min _{y: x \leq y \leq x+w}\{L(y)\}=L(x)$, which implies that the optimal production quantity is zero. When $\hat{y} \geq x$, we can write $H(w \mid x)$ using Equations (2) and (A1) as follows.

Case I: $\quad(0 \leq w \leq U)$

$$
H(w \mid x)= \begin{cases}L(x+w), & \text { if } w \leq \hat{y}-x, \\ L(\hat{y}), & \text { if } \hat{y}-x<w .\end{cases}
$$

Case II: $\quad(U<w \leq U+V)$

$$
H(w \mid x)= \begin{cases}(w-U) c_{\mathrm{u}}+L(x+w), & \text { if } w \leq \hat{y}-x \\ (w-U) c_{\mathrm{u}}+L(\hat{y}), & \text { if } \hat{y}-x<w .\end{cases}
$$

Case III: $(U+V<w)$

$$
H(w \mid x)= \begin{cases}V c_{\mathrm{u}}+p\left((w-U-V) c_{\mathrm{tw}}+L(x+w)\right)+(1-p) L(x+U+V), & \text { if } w \leq \hat{y}-x, \\ V c_{\mathrm{u}}+p\left((w-U-V) c_{\mathrm{tw}}+L(\hat{y})\right)+(1-p) L(\psi(x)), & \text { if } \hat{y}-x<w,\end{cases}
$$

where

$$
\psi(x)= \begin{cases}x+U+V, & \text { if } x \leq \hat{y}-U-V \\ \hat{y}, & \text { otherwise }\end{cases}
$$

Note that $\psi(x)$ is constant in $w$. Therefore, $H(w \mid x)$ is convex in $w$ in all of the above regions, for all values of $x$, which follows from the convexity of $L(\cdot)$. The first derivative of $H(w \mid x)$ in the second region of Case III is $\mathrm{d} H(w \mid x) / \mathrm{d} w=p c_{\mathrm{tw}}$, which is always positive. This indicates that the optimal value of $w$ cannot reside in this region and hence it is never optimal to order more than necessary.

To conclude the convexity of $H(w \mid x)$ we need to show that convexity is preserved in transition points $w=U$ and $w=U+V$. We denote the respective regions by the following subscripts: I $(0 \leq w \leq U)$, II $(U<w \leq U+V)$ and III $(U+V<w)$. The following first-order condition is sufficient:

$$
\frac{\mathrm{d} H_{\mathrm{I}}(w \mid x)}{\mathrm{d} w} \leq \frac{\mathrm{d} H_{\mathrm{II}}(w \mid x)}{\mathrm{d} w} \leq \frac{\mathrm{d} H_{\mathrm{III}}(w \mid x)}{\mathrm{d} w} .
$$

For the first transition point we need to check the above inequalities for values of $x$ below and above $\hat{y}-U$. If $x+U \leq \hat{y}$ then we have $\lim _{w \rightarrow U^{-}} \mathrm{d} H_{\mathrm{I}}(w \mid x) / \mathrm{d} w=L^{\prime}(x+U)$ from the first region of Case I, and $\lim _{w \rightarrow U^{+}} \mathrm{d} H_{\mathrm{II}}(w \mid x) / \mathrm{d} w=c_{\mathrm{u}}+L^{\prime}(x+U)$ from the first region of Case II. If $x+U \geq \hat{y}$ then we have $\lim _{w \rightarrow U^{-}} \mathrm{d} H_{\mathrm{I}}(w \mid x) / \mathrm{d} w=0$ from the second region of Case $\mathrm{I}$, and $\lim _{w \rightarrow U^{+}} \mathrm{d} H_{\mathrm{II}}(w \mid x) / \mathrm{d} w=c_{\mathrm{u}}$ from the second region of Case II. Since $c_{\mathrm{u}}>0$, the convexity is preserved at the junction point $U$. If $x+U+V \leq \hat{y}$ then we have $\lim _{w \rightarrow(U+V)^{-}} \mathrm{d} H_{\text {II }}(w \mid x) / \mathrm{d} w=$ $c_{\mathrm{u}}+L^{\prime}(x+U+V)$ from the first region of Case II, and $\lim _{w \rightarrow\left(U+V V^{+}\right.} \mathrm{d} H_{\mathrm{III}}(w \mid x) / \mathrm{d} w=$ $p c_{\mathrm{tw}}+p L^{\prime}(x+U+V)$ from the first region of Case III. If $x+U+V \geq \hat{y}$ then we have $\lim _{w \rightarrow(U+V)^{-}} \mathrm{d} H_{\mathrm{I}}(w \mid x) / \mathrm{d} w=c_{\mathrm{u}}$ from the second region of Case II, and $\lim _{w \rightarrow(U+V)^{+}} \mathrm{d} H_{\mathrm{II}}(w \mid x) / \mathrm{d} w=p c_{\mathrm{tw}}$ from the second region of Case III. If $p c_{\mathrm{tw}}>c_{\mathrm{u}}$, the convexity is preserved at the junction point $U+V$ since $L(x+U+V)<0$ when $x+U+V \leq \hat{y}$. 
If $\hat{y}-U \leq x<\hat{y}$ then the value of $w$ minimising $H(w \mid x)$ is in region (I) and it is the minimiser of $L(x+w)$. From the classical newsboy solution we derive the optimal requested capacity target as

$$
w^{*}(x)=\hat{y}-x=G^{-1}\left(\frac{b}{h+b}\right)-x=y_{\mathrm{p}}^{*}-x .
$$

We let $y_{\mathrm{p}}^{*}=\hat{y}$. If $y_{\mathrm{p}}^{*}-U-V<x<y_{\mathrm{p}}^{*}-U$, then the minimiser of the function $H(w \mid x)$ is in region (II). From the first-order condition, we have

$$
0=c_{\mathrm{u}}+L^{\prime}(x+w) \text {. }
$$

Note that the optimality equation may not be satisfied even if $x$ is in the above region, particularly if $c_{\mathrm{u}}+L^{\prime}(x+w)>0$. In that case the optimal policy is to produce at full permanent capacity, $w^{*}=U$, and the resulting order-up-to level is $x+U$. Otherwise, using the solution of the optimality equation the optimal requested capacity is found to be $w^{*}(x)=G^{-1}\left(\left(b-c_{\mathrm{u}}\right) /(b+h)\right)-x$ and the corresponding order-up-to level is $y_{\mathrm{v}}^{*}=G^{-1}\left(\left(b-c_{\mathrm{u}}\right) /(b+h)\right)$. Note that, for non-negative $c_{\mathrm{u}}, y_{\mathrm{v}}^{*} \geq y_{\mathrm{p}}^{*}$. The optimal capacity policy for this particular region is

$$
w^{*}(x)= \begin{cases}y_{\mathrm{v}}^{*}-x, & \text { if } x \leq y_{\mathrm{v}}^{*}-U, \\ U, & \text { if } y_{\mathrm{v}}^{*}-U \leq x<y_{\mathrm{p}}^{*}-U .\end{cases}
$$

If $x<y_{\mathrm{v}}^{*}-U-V$ then the minimiser of $H(w \mid x)$ is in region III. Similarly, we obtain

$$
w^{*}(x)= \begin{cases}y_{\mathrm{c}}^{*}-x, & \text { if } x \leq y_{\mathrm{c}}^{*}-U-V, \\ U+V, & \text { if } y_{\mathrm{c}}^{*}-U-V \leq x<y_{\mathrm{v}}^{*}-U-V,\end{cases}
$$

where $y_{\mathrm{c}}^{*}=G^{-1}\left(\left(b-c_{\mathrm{tw}}\right) /(b+h)\right)$. For $0<c_{\mathrm{u}}<c_{\mathrm{tw}}$, the optimal values for the above functions have the following relation: $y_{\mathrm{p}}^{*}>y_{\mathrm{v}}^{*}>y_{\mathrm{c}}^{*}$. Using the above property, the single-period state-dependent order-up-to can be written as

$$
y^{*}(x)= \begin{cases}y_{\mathrm{c}}^{*}, & \text { if } x<y_{\mathrm{c}}^{*}-U-V, \\ x+U+V, & \text { if } y_{\mathrm{c}}^{*}-U-V<x \leq y_{\mathrm{v}}^{*}-U-V, \\ y_{\mathrm{v}}^{*}, & \text { if } y_{\mathrm{v}}^{*}-U-V<x \leq y_{\mathrm{v}}^{*}-U, \\ x+U, & \text { if } y_{\mathrm{v}}^{*}-U<x \leq y_{\mathrm{p}}^{*}-U, \\ y_{\mathrm{p}}^{*}, & \text { if } y_{\mathrm{p}}^{*}-U<x \leq y_{\mathrm{p}}^{*}, \\ x, & \text { if } y_{\mathrm{p}}^{*}<x,\end{cases}
$$

and $w^{*}(x)=y^{*}(x)-x$.

Using $y^{*}(x)$ we can write $f(x)$ as

$$
f(x)=U c_{\mathrm{p}}+V c_{\mathrm{r}}+ \begin{cases}V c_{\mathrm{u}}+p\left(c_{\mathrm{tw}}\left(y_{\mathrm{c}}^{*}-U-V-x\right)\right. & \\ \left.\quad+L\left(y_{\mathrm{c}}^{*}\right)\right)+(1-p) L(x+U+V), & \text { if } x<y_{\mathrm{c}}^{*}-U-V, \\ V c_{\mathrm{u}}+L(x+U+V), & \text { if } y_{\mathrm{c}}^{*}-U-V<x \leq y_{\mathrm{v}}^{*}-U-V, \\ \left(y_{\mathrm{v}}^{*}-U-x\right) c_{\mathrm{u}}+L\left(y_{\mathrm{v}}^{*}\right), & \text { if } y_{\mathrm{v}}^{*}-U-V<x \leq y_{\mathrm{v}}^{*}-U, \\ L(x+U), & \text { if } y_{\mathrm{v}}^{*}-U<x \leq y_{\mathrm{p}}^{*}-U, \\ L\left(y_{\mathrm{p}}^{*}\right), & \text { if } y_{\mathrm{p}}^{*}-U<x \leq y_{\mathrm{p}}^{*}, \\ L(x), & \text { if } y_{\mathrm{p}}^{*}<x .\end{cases}
$$

Similar to the convexity of the function $H$, it can be shown that $f(x)$ is also convex. Then the theorem can be proved by regular inductive arguments. For details, see Pac (2006).

Proof (Proof of Theorem 4.3): We prove by induction. Recall from (A2) that the optimal orderup-to levels of the last period are all independent of $p$ and hence are non-increasing in $p$. For any 
period $t$, we have

$$
H_{t}(w \mid x)= \begin{cases}\min _{y: x \leq y \leq x+w}\left\{J_{t}(y)\right\}, & \text { if } 0 \leq w \leq U, \\ (w-U) c_{\mathrm{u}}+\min _{y: x \leq y \leq x+w}\left\{J_{t}(y)\right\}, & \text { if } U<w \leq U+V, \\ V c_{\mathrm{u}}+p\left(c_{\mathrm{tw}}(w-U-V)+\min _{y: x \leq y \leq x+w}\left\{J_{t}(y)\right\}\right) & \\ \quad+(1-p) \min _{y: x \leq y \leq x+U+V}\left\{J_{t}(y)\right\}, & \text { if } U+V<w,\end{cases}
$$

where $J_{t}(y)=L(y)+\alpha E f_{t+1}(y-Z)$. As shown in the proof of Theorem 4.1, the critical order-up-to levels $y_{t p}^{*}, y_{t v}^{*}$, and $y_{t c}^{*}$ are found by equating the following expressions to zero in the regions specified, respectively, when $w$ is set to $y-x$ :

$$
\frac{\mathrm{d} H_{t}\left(y_{t} \mid x\right)}{\mathrm{d} y}= \begin{cases}L^{\prime}(y)+\alpha \frac{\mathrm{d} E\left[f_{t+1}(y-z)\right]}{\mathrm{d} y}, & \text { if } y_{t} \leq x+U, \\ c_{\mathrm{u}}+L^{\prime}(y)+\alpha \frac{\mathrm{d} E\left[f_{t+1}(y-z)\right]}{\mathrm{d} y}, & \text { if } x+U \leq y_{t} \leq x+U+V, \\ p\left[c_{\mathrm{tw}}+L^{\prime}(y)+\alpha \frac{\mathrm{d} E\left[f_{t+1}(y-z)\right]}{\mathrm{d} y}\right], & \text { if } x+U+V \leq y_{t} .\end{cases}
$$

With the inductive assumption, we assume that the optimal order-up-to level, $y_{t+1}(x)$, for period $t+1$ is non-increasing in $p$. Since $H_{t+1}(y \mid x)$ is convex in $y$, our inductive assumption implies that $\mathrm{d} H_{t+1}\left(y_{t+1} \mid x\right) / \mathrm{d} y$ is non-decreasing in $p$, which gives the following:

$$
\frac{\mathrm{d}^{2} H_{t+1}\left(y_{t+1} \mid x\right)}{\mathrm{d} y \mathrm{~d} p}=\frac{\mathrm{d}^{2} E\left[f_{t+2}(y-z)\right]}{\mathrm{d} y \mathrm{~d} p} \geq 0
$$

To complete the proof we analyse Equation (A4), the first-order condition for period $t$, and show that it is non-decreasing in $p$. Notice that the only term that can depend on the value of $p$ in the firstorder condition is $\mathrm{d} E[f(y-z)] / \mathrm{d} y$. Therefore, we analyse this part and show that it is increasing in $p$. Let $y_{\mathrm{p}}^{*}, y_{\mathrm{v}}^{*}$ and $y_{\mathrm{c}}^{*}$ be the points where the first-order condition is satisfied for production with permanent capacity only, permanent plus contracted capacity and production with temporary capacity, at period $t+1$

$$
\begin{aligned}
E\left[f_{t+1}(y-z)\right]= & \int_{0}^{y-y_{\mathrm{p}}^{*}} J_{t+1}(y-z) f(z) \mathrm{d} z+\int_{y-y_{\mathrm{p}}^{*}}^{y-y_{\mathrm{p}}^{*}+U} J_{t+1}\left(y_{\mathrm{p}}^{*}\right) f(z) \mathrm{d} z \\
& +\int_{y-y_{\mathrm{p}}^{*}+U}^{y-y_{\mathrm{v}}^{*}+U} J_{t+1}(y-z+U) f(z) \mathrm{d} z+\int_{y-y_{\mathrm{v}}^{*}+U}^{y-y_{\mathrm{v}}^{*}+U+V}\left[c_{\mathrm{u}}\left(y_{\mathrm{v}}^{*}-y+z-U\right)+J_{t+1}\left(y_{\mathrm{v}}^{*}\right)\right] f(z) \mathrm{d} z \\
& +\int_{y-y_{\mathrm{v}}^{*}+U+V}^{y-y_{\mathrm{c}}^{*}+U+V}\left[c_{\mathrm{u}}(V)+J_{t+1}(y-z+U+V)\right] f(z) \mathrm{d} z \\
& +\int_{y-y_{\mathrm{c}}^{*}+U+V}^{\infty}\left[c_{\mathrm{u}}(V)+(1-p)\left(J_{t+1}(y-z+U+V)\right)\right. \\
& \left.+p\left\{c_{(t+1) w}\left(y_{\mathrm{c}}^{*}-y+z-U-V\right)+J_{t+1}\left(y_{\mathrm{c}}^{*}\right)\right\}\right] f(z) \mathrm{d} z,
\end{aligned}
$$

where $J_{t+1}(y)=L(y)+\alpha E\left[f_{t+2}(y-z)\right]$. If we differentiate this expectation with respect to $y$ we obtain the following expression:

$$
\begin{aligned}
\frac{\mathrm{d} E[f(y-z)]}{\mathrm{d} y}= & \int_{0}^{y-y_{\mathrm{p}}^{*}} J_{t+1}^{\prime}(y-z) f(z) \mathrm{d} z+\int_{y-y_{\mathrm{p}}^{*}+U}^{y-y_{v}^{*}+U} J_{t+1}^{\prime}(y-z+U) f(z) \mathrm{d} z \\
& -\int_{y-y_{v}^{*}+U}^{y-y_{v}^{*}+U+V} c_{\mathrm{u}} f(z) \mathrm{d} z+\int_{y-y_{\mathrm{v}}^{*}+U+V}^{y-y_{\mathrm{c}}^{*}+U+V} J_{t+1}^{\prime}(y-z+U+V) f(z) \mathrm{d} z \\
& +\int_{y-y_{\mathrm{c}}^{*}+U+V}^{\infty}\left[(1-p) J_{t+1}^{\prime}(y-z+U+V)-p c_{(t+1) w}\right] f(z) \mathrm{d} z .
\end{aligned}
$$


To analyse how this expression changes with respect to $p$, we differentiate the above expression with respect to $p$ :

$$
\begin{aligned}
& \frac{\mathrm{d}^{2} E[f(y-z)]}{\mathrm{d} y \mathrm{~d} p}=-\frac{\mathrm{d} y_{\mathrm{v}}^{*}}{\mathrm{~d} p} f\left(y-y_{\mathrm{v}}^{*}+U\right)\left[c_{\mathrm{u}}+J_{t+1}^{\prime}\left(y_{\mathrm{v}}^{*}\right)\right]+\frac{\mathrm{d} y_{\mathrm{v}}^{*}}{\mathrm{~d} p} f\left(y-y_{\mathrm{v}}^{*}+U+V\right)\left[c_{\mathrm{u}}+J_{t+1}^{\prime}\left(y_{\mathrm{v}}^{*}\right)\right] \\
& +\frac{\mathrm{d} y_{\mathrm{c}}^{*}}{\mathrm{~d} p} f\left(y-y_{\mathrm{c}}^{*}+U+V\right)\left[-p J_{t+1}^{\prime}\left(y_{\mathrm{c}}^{*}\right)+p c_{(t+1) w}\right] \\
& -\int_{y-y_{\mathrm{c}}^{*}+U+V}^{\infty}\left[c_{(t+1) w}+J_{t+1}^{\prime}(y-z+U+V)\right] f(z) \mathrm{d} z \\
& +\int_{0}^{y-y_{\mathrm{p}}^{*}} \frac{\mathrm{d}^{2} J_{t+1}(y-z)}{\mathrm{d} y \mathrm{~d} p} f(z) \mathrm{d} z+\int_{y-y_{\mathrm{p}}^{*}+U}^{y-y_{\mathrm{v}}^{*}+U} \frac{\mathrm{d}^{2} J_{t+1}(y-z+U)}{\mathrm{d} y \mathrm{~d} p} f(z) \mathrm{d} z \\
& +\int_{y-y_{v}^{*}+U+V}^{y-y_{\mathrm{c}}^{*}+U+V} \frac{\mathrm{d}^{2} J_{t+1}(y-z+U+V)}{\mathrm{d} y \mathrm{~d} p} f(z) \mathrm{d} z \\
& +\int_{y-y_{\mathrm{c}}^{*}+U+V}^{\infty}(1-p) \frac{\mathrm{d}^{2} J_{t+1}(y-z+U+V)}{\mathrm{d} y \mathrm{~d} p} f(z) \mathrm{d} z \\
& =-\int_{y-y_{\mathrm{c}}^{*}+U+V}^{\infty}\left[c_{(t+1) w}+J_{t+1}^{\prime}(y-z+U+V)\right] f(z) \mathrm{d} z \\
& +\int_{0}^{y-y_{\mathrm{p}}^{*}} \frac{\mathrm{d}^{2} E\left[f_{t+2}(y-z)\right]}{\mathrm{d} y \mathrm{~d} p} f(z) \mathrm{d} z+\int_{y-y_{\mathrm{p}}^{*}+U}^{y-y_{\mathrm{v}}^{*}+U} \frac{\mathrm{d}^{2} E\left[f_{t+2}(y-z+U)\right]}{\mathrm{d} y \mathrm{~d} p} f(z) \mathrm{d} z \\
& +\int_{y-y_{v}^{*}+U+V}^{y-y_{\mathrm{c}}^{*}+U+V} \frac{\mathrm{d}^{2} E\left[f_{t+2}(y-z+U+V)\right]}{\mathrm{d} y \mathrm{~d} p} f(z) \mathrm{d} z \\
& +\int_{y-y_{\mathrm{c}}^{*}+U+V}^{\infty}(1-p) \frac{\mathrm{d}^{2} E\left[f_{t+2}(y-z+U+V)\right]}{\mathrm{d} y \mathrm{~d} p} f(z) \mathrm{d} z \\
& \geq 0 \text {. }
\end{aligned}
$$

Notice that the first three terms in the initial identity are all zero, which follows from the first-order condition. The final term is always positive as $y-z+U+V \leq y_{\mathrm{c}}^{*}$, therefore the term $c_{(t+1) w}+J_{t+1}^{\prime}(y-z+U+V)$ is always negative in the region of integration, and from the inductive assumption $\mathrm{d}^{2} E\left(f_{t+2}(y-\mathrm{z})\right) / \mathrm{d} y, \mathrm{~d} p$ is always positive, therefore the corresponding integrals are all non-negative, which gives us the desired result.

Proof (Proof of Theorem 5.1): The theorem is proved for a continuous uniform distribution. Omitting the constant term $U c_{\mathrm{p}}+V c_{\mathrm{r}}$, the single-period cost function for the uniform temporary labour uncertainty case can be written as

$$
H(w \mid x)= \begin{cases}\varphi(w \mid x), & \text { if } 0 \leq w \leq U, \\ (w-U) c_{\mathrm{u}}+\varphi(w \mid x), & \text { if } U<w \leq U+V, \\ V c_{\mathrm{u}}+\int_{0}^{w-U-V}\left(m c_{\mathrm{tw}}+\varphi(U+V+m \mid x)\right) \frac{1}{w-U-V} \mathrm{~d} m, & \text { if } U+V<w .\end{cases}
$$

To prove that $H(w \mid x)$ is convex it is sufficient to analyse the case with a temporary capacity region and the corresponding transition point, since for $w \leq U+V$ the function remains identical for all labour supply uncertainty types. 
For $w>U+V$ :

$$
H(w \mid x)= \begin{cases}V c_{\mathrm{u}}+c_{\mathrm{tw}} \frac{(w-U-V)}{2}+\int_{0}^{w-U-V} \frac{L(x+U+V+m)}{(w-U-V)} \mathrm{d} m, & \text { if } U+V<w \leq y_{\mathrm{p}}^{*}-x, \\ V c_{\mathrm{u}}+c_{\mathrm{tw}} \frac{(w-U-V)}{2}+\int_{0}^{y_{\mathrm{p}}^{*}-x-U-V} \frac{L(x+U+V+m)}{(w-U-V)} \mathrm{d} m & \\ \quad+\int_{y_{\mathrm{p}}^{*}-x-U-V}^{w-U-V} \frac{L\left(y_{\mathrm{p}}^{*}\right)}{(w-U-V)} \mathrm{d} m, & \text { if } y_{\mathrm{p}}^{*}-x<w .\end{cases}
$$

We take the first derivative of the function to check the first-order condition:

$$
\frac{\mathrm{d} H(w \mid x)}{\mathrm{d} w}= \begin{cases}\frac{c_{\mathrm{tw}}}{2}+\frac{L(x+w)}{(w-U-V)}-\int_{0}^{w-U-V} \frac{L(x+U+V+m)}{(w-U-V)^{2}} \mathrm{~d} m, & \text { if } U+V<w \leq y_{\mathrm{p}}^{*}-x, \\ \frac{c_{\mathrm{tw}}}{2}+\frac{L\left(y_{\mathrm{p}}^{*}\right)\left(y_{\mathrm{p}}^{*}-x-U-V\right)}{(w-U-V)^{2}}-\int_{0}^{y_{\mathrm{p}}^{*}-x-U-V} \frac{L(x+U+V+m)}{(w-U-V)^{2}} \mathrm{~d} m, & \text { if } y_{\mathrm{p}}^{*}-x<w .\end{cases}
$$

At the transition point $w=y_{\mathrm{p}}^{*}-x$ the first derivatives are equal, therefore if the second derivative is non-negative on both sides of the transition point, the first-order condition will be satisfied.

$$
\frac{\mathrm{d}^{2} H(w \mid x)}{\mathrm{d} w^{2}}= \begin{cases}\frac{L^{\prime}(x+w)(w-U-V)^{2}-2 L(x+w)(w-U-V)}{(w-U-V)^{3}} & \text { if } U+V<w \leq y_{\mathrm{p}}^{*}-x . \\ \frac{2 \int_{0}^{w-U-v} L(x+U+V+m) \mathrm{d} m}{(w-U-V)^{3}}, & \\ \frac{2 \int_{0}^{y_{\mathrm{p}}^{*}-x-U-V} L(x+U+V+m) \mathrm{d} m}{(w-U-V)^{3}}-\frac{2 L\left(y_{\mathrm{p}}^{*}\right)\left(y_{\mathrm{p}}^{*}-x-U-V\right)}{(w-U-V)^{3}}, & \text { if } y_{\mathrm{p}}^{*}-x<w .\end{cases}
$$

It is evident that $\mathrm{d}^{2} H(w \mid x) / \mathrm{d} w^{2}$ is positive for $w>y_{\mathrm{p}}^{*}-x$, because the first term in the nominator is greater than the second term, since it integrates $L(x+U+V+m)$ over a region where the values are greater than the optimal $L\left(y_{\mathrm{p}}^{*}\right)$, whereas the second term is equivalent to the integration of $L\left(y_{\mathrm{p}}^{*}\right)$ over the same region. For $U+V<w \leq y_{\mathrm{p}}^{*}-x$ we take the limit of the second derivative as $w \rightarrow U+V$ and show that it is positive, and remains positive throughout the whole domain:

$$
\lim _{w \rightarrow U+V} \frac{L^{\prime}(x+w)(w-U-V)^{2}-2 L(x+w)(w-U-V)+2 \int_{0}^{w-U-v} L(x+U+V+m) \mathrm{d} m}{(w-U-V)^{3}}=\frac{0}{0} .
$$

Using L'Hopital's rule we obtain

$$
\begin{aligned}
& \frac{L^{\prime \prime}(x+w)(w-U-V)^{2}+2 L^{\prime}(x+w)(w-U-V)-2 L^{\prime}(x+w)(w-U-V)-2 L(x+w)+2 L(x+w)}{3(w-U-V)^{2}} \\
& \quad=\frac{L^{\prime \prime}(x+w)}{3}>0 .
\end{aligned}
$$

The second derivative is positive at $U+V$, and we have to ensure that it remains positive for $w>U+V$. To do so we check the numerator of the second derivative, since the denominator is always positive for $w>U+V$. We take the derivative of the numerator and check if it is positive. Let us denote the numerator by $\varpi(w)$, then $\mathrm{d} \varpi(w) / \mathrm{d} w=L^{\prime \prime}(x+w)(w-U-V)^{2}$, which is positive for all $w$, hence the function is convex for $w>U+V$.

To conclude the convexity of $H(w \mid x)$, we need to show that the convexity is preserved at the transition point $w=U+V$. Since $H(w \mid x)$ is dependent on the starting inventory level $x$, the first-order condition should be satisfied for all $x$. It is sufficient to analyse the transition point for $x<y_{\mathrm{p}}^{*}-U-V$ and $x \geq y_{\mathrm{p}}^{*}-U-V$. Note that we analyse the derivative of the function on 
both sides of the transition point. For the initial case, $H(w \mid x)$ takes the following form near the transition point:

$$
H(w \mid x)= \begin{cases}c_{\mathrm{u}}(w-U)+L(x+w), & \text { if } U<w \leq U+V, \\ V c_{\mathrm{u}}+c_{\mathrm{tw}} \frac{w-U-V}{2}+\int_{0}^{w-U-V} \frac{L(x+U+V+m)}{w-U-V} \mathrm{~d} m, & \text { if } U+V<w \leq y_{\mathrm{p}}^{*}-x .\end{cases}
$$

The first derivative for this region is

$$
\frac{\mathrm{d} H(w \mid x)}{\mathrm{d} w}= \begin{cases}c_{\mathrm{u}}+L^{\prime}(x+w), & \text { if } U<w \leq U+V, \\ \frac{c_{\mathrm{tw}}}{2}+\frac{L(x+w)}{w-U-V}+\int_{0}^{w-U-V} \frac{L(x+U+V+m)}{(w-U-V)^{2}} \mathrm{~d} m, & \text { if } U+V<w \leq y_{\mathrm{p}}^{*}-x .\end{cases}
$$

The first-order condition for the above region is

$$
c_{\mathrm{u}}+L^{\prime}(x+w) \leq \frac{c_{\mathrm{tw}}}{2}+\frac{L(x+w)(w-U-v)-\int_{0}^{w-U-V} L(x+U+V+m) \mathrm{d} m}{(w-U-V)^{2}} .
$$

Taking the limit as $w \rightarrow U+V$ we obtain

$$
c_{\mathrm{u}}+L^{\prime}(x+w) \leq \frac{c_{\mathrm{tw}}}{2}+\frac{L^{\prime}(x+w)}{2} .
$$

Note that the above inequality holds if $c_{\mathrm{u}} \leq c_{\mathrm{tw}} / 2$.

For the second case $\left(x \geq y_{\mathrm{p}}^{*}-U-V\right)$, the cost function takes the following form:

$$
H(w \mid x)= \begin{cases}c_{\mathrm{u}}(w-U)+L\left(y_{\mathrm{p}}^{*}\right), & \text { if } y_{\mathrm{p}}^{*}-x<w \leq U+V, \\ V c_{\mathrm{u}}+c_{\mathrm{tw}} \frac{w-U-V}{2}+L\left(y_{\mathrm{p}}^{*}\right), & \text { if } U+V<w .\end{cases}
$$

The first derivative for this region is in the following form:

$$
\frac{\mathrm{d} H(w \mid x)}{\mathrm{d} w}= \begin{cases}c_{\mathrm{u}}, & \text { if } y_{\mathrm{p}}^{*}-x<w \leq U+V, \\ \frac{c_{\mathrm{tw}}}{2}, & \text { if } U+V<w .\end{cases}
$$

For the first-order condition to hold, $c_{\mathrm{u}}$ must be less than or equal to $c_{\mathrm{tw}} / 2$. Therefore, if $c_{\mathrm{u}} \leq c_{\mathrm{tw}} / 2$ then the single-period cost function is convex in $w$ for all starting inventory levels $x$. 